\title{
Entropy-based study of imaging quality in holographic optical elements
}

\author{
L. Carretero and A. Fimia \\ Laboratorio de Optica, Departamento Interuniversitario de Optica, Universidad de Alicante, Apartado 99, Alicante E 03080, Spain
}

\section{A. Beléndez}

Departamento de Ingeniería de Sistemas y Comunicaciones, Universidad de Alicante, Apartado 99, Alicante E 03080, Spain

Received March 7, 1994

A method for obtaining the best image plane for holographic optical elements by the use of the concept of entropy is described. This method is applied to in-line holographic lenses with different values of spherical aberration. Numerical results show that for holograms with large aberrations the best image plane (obtained by the use of the concept of entropy) is different from the minimum-aberration-variance plane.

Recently the concept of entropy has been used in optics to locate coherent propagation planes that are interesting from an optical viewpoint, ${ }^{1}$ and the study of the two-image depth-from-focus problem has been considered in terms of entropy loss in a linear filter. ${ }^{2}$ In digital image processing the concept of entropy has been used as a means of recovering pictorial information in degraded photographs., ${ }^{3,4}$ The formation of images with incoherent light, ${ }^{5}$ the study of partial polarization, and coherence theory ${ }^{6}$ are other fields in which an entropic method has been used. Dähne and Lanzl ${ }^{7}$ have described an automatic focusing technique for a computer-controlled optical microscope based on the concepts of entropy. Other maximum principles have been used in image restoration. ${ }^{8}$

In this Letter we suggest the use of the concept of entropy to locate the position of the best image plane for a holographic optical element (HOE) in the presence not only of small but also of large aberrations. Let us outline the properties of hologram aberration derived by Champagne. ${ }^{9}$ We assume that the HOE is located on the $X Y$ plane and that the point source $Q\left(x_{q}, y_{q}, z_{q}\right)$ of a spherical wave is defined in terms of the parameters $R_{q}, \alpha_{q}$, and $\beta_{q}$, as can be seen in Fig. 1 of Ref. 10. Here $q=\mathrm{r}, 0$, c, and i denote the reference, object, reconstruction, and image points, respectively. The recording and the reconstruction wavelengths are $\lambda_{\mathrm{r}}$ and $\lambda_{\mathrm{c}}$, respectively. According to Refs. 9, 11, and 12 the phase aberration at a point $(x, y)$ in the exit pupil plane $\Delta(x, y)$ is given by

$$
\Delta(x, y)=\phi_{\mathrm{c}}(x, y)-\phi_{\mathrm{i}}(x, y) \pm\left[\phi_{0}(x, y)-\phi_{\mathrm{r}}(x, y)\right]
$$

the \pm refers to the positive and the negative first diffraction orders from the hologram, and $\phi_{q}$ is the phase of a spherical wave. Its value in the plane of the hologram is ${ }^{12}$

$$
\phi_{q}(x, y)=\frac{2 \pi}{\lambda_{q}}\left[r_{q}(x, y)-R_{q}\right]
$$

The phase aberration $\Delta$ is related to the wave aberration $W$ according to $\Delta=2\left(2 \pi / \lambda_{\mathrm{c}}\right) W$. Using Eqs. (1) and (2) we can write $W(x, y)$ as

$$
W=r_{\mathrm{c}}-r_{\mathrm{i}} \pm \mu\left(r_{\mathrm{o}}-r_{\mathrm{r}}\right)-\left[R_{\mathrm{c}}-R_{\mathrm{i}} \pm \mu\left(R_{\mathrm{o}}-R_{\mathrm{r}}\right)\right],
$$

where $\mu$ denotes the wavelength shift $\lambda_{\mathrm{c}} \lambda_{\mathrm{r}}$. The distance $R_{g}$ and the angle $\alpha_{g}$ of the Gaussian image point are given by ${ }^{9}$

$$
\begin{aligned}
\frac{1}{R_{g}} & =\frac{1}{R_{\mathrm{c}}} \pm \mu\left(\frac{1}{R_{\mathrm{o}}}-\frac{1}{R_{\mathrm{r}}}\right), \\
\sin \alpha_{g} & =\sin \alpha_{\mathrm{c}} \pm \mu\left(\sin \alpha_{\mathrm{o}}-\sin \alpha_{\mathrm{r}}\right) .
\end{aligned}
$$

Equation (4) defines the radius $R_{g}$ of the reference sphere centered on the Gaussian image point, G. We introduce a local coordinate frame $X^{\prime} Y^{\prime} Z^{\prime}$ fixed to point $\mathrm{G}$ as the origin. The $Z^{\prime}$ axis is defined by the principal ray that runs from the center of the hologram to Gaussian image point G. For simplicity we choose the $X^{\prime} Y^{\prime}$ plane as the image plane so that $\left(x^{\prime}, y^{\prime}\right)$ are coordinates of an image point in this plane, and the coordinates of this image point in the $X Y Z$ coordinates system are $\left(x_{\mathrm{i}}, y_{\mathrm{i}}, z_{\mathrm{i}}\right)$. The intensity of the image in a plane normal to the chief ray ( $Z^{\prime}$ axis) at a distance $z^{\prime}$ from the center of the HOE may be written as ${ }^{13}$

$$
\begin{aligned}
& I\left(x^{\prime}, y^{\prime} ; z^{\prime}\right) \\
& =\frac{1}{B^{2}}\left|\iint_{S} A(x, y) \exp \left[i \Delta\left(x, y ; x^{\prime}, y^{\prime} ; z^{\prime}\right)\right] \mathrm{d} x \mathrm{~d} y\right|^{2},
\end{aligned}
$$

where $A(x, y)=1$ (uniform amplitude) and $S$ represents the area of the exit pupil where the integration is done. In Eq. (6), $B$ is the amplitude at the Gaussian image point $\left(x^{\prime}=y^{\prime}=0\right)$ in the absence of aberrations. Intensity can be interpreted as a probability-density function, provided that it is adequately normalized ${ }^{3,4}$ as 


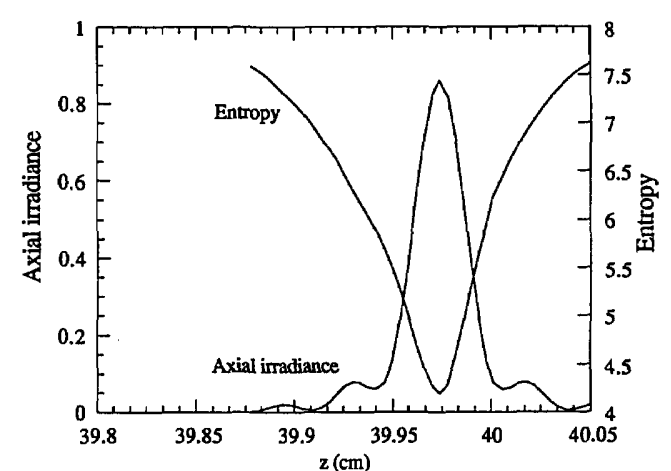

(a)

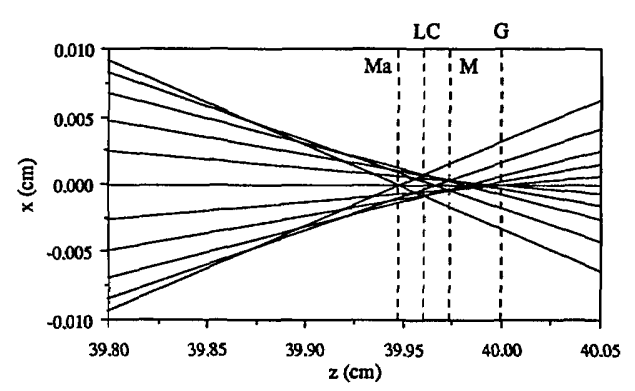

(b)

Fig. 1. (a) Entropy and axial irradiance for holographic lenses with a diameter of $5 \mathrm{~cm}$, (b) ray tracing: $\mathrm{Ma}$, marginal plane; LC, last confusion plane; M, medium plane (minimum aberration variance); G, Gaussian plane.

$$
P\left(x^{\prime}, y^{\prime} ; z^{\prime}\right)=\frac{I\left(x^{\prime}, y^{\prime} ; z^{\prime}\right)}{\iint_{A^{\prime}} I\left(x^{\prime}, y^{\prime} ; z^{\prime}\right) \mathrm{d} x^{\prime} \mathrm{d} y^{\prime}},
$$

where $A^{\prime}$ is the integration area in the image plane. For an image plane situated at a $z^{\prime}$ position, we can define the entropy of the image formed on this plane as

$$
S\left(z^{\prime}\right)=-\iint_{A^{\prime}} P\left(x^{\prime}, y^{\prime} ; z^{\prime}\right) \ln \left[P\left(x^{\prime}, y^{\prime} ; z^{\prime}\right)\right] \mathrm{d} x^{\prime} \mathrm{d} y^{\prime},
$$

and numerical calculations are necessary for obtaining this entropy. In this case, entropy should be interpreted as a property of the way in which energy is distributed on an image plane intensity, and, in this sense, entropy gives us a measure of the aberrations in the considered plane. The lowest entropy plane will be the best image plane in the sense of minimal aberrations. In the presence of aberrations this plane is different from the plane that contains the Gaussian image point. The plane with the lowest entropy is chosen as the best image plane. For an optical system with a circular exit pupil, the aberration-free irradiance distribution is the Airy pattern, and it is centered at the Gaussian image point. The quality of the image is limited only by the diffraction of the object radiation at the exit pupil of the system. Then the value for the entropy obtained for this image plane will be the lowest value of the entropy for this optical system. To illustrate our entropy-based study of quality images in HOE's, we use the above-mentioned definition of entropy to obtain the best image plane for two in-line holographic lenses with spherical aberration. To illustrate our method, from Eq. (8) we numerically evaluate the entropy along the $Z$ axis for two holographic lenses with diameters $D=5 \mathrm{~cm}$ and $D=8 \mathrm{~cm}$ recorded with a divergent spherical wave whose curvature radius is $R_{\mathrm{r}}=-120 \mathrm{~cm}$ and with a convergent spherical wave with the source point separated $R_{0}=60 \mathrm{~cm}$ from the hologram center with a recording wavelength of $\lambda_{\mathrm{r}}=633 \mathrm{~nm}$. The two source points are situated on the axis normal to the holographic plate. The hologram is reconstructed with a collimated beam $\left(R_{\mathrm{c}}=\infty\right)$ and the wavelength used in the recording step. Using Eq. (4), we obtain the Gaussian point position that is situated $40 \mathrm{~cm}$ from the HOE. Axial irradiance is also numerically evaluated from Eq. (6), with $x^{\prime}=y^{\prime}=0$.

When ray aberrations are considered, it is possible to mix one aberration with another in order to minimize the size of the ray spot on an image plane. In the case of spherical aberration, it is possible to balance the effect of spherical aberration by observing the image not on the Gaussian plane but on a defocused image plane, and the circle of least confusion is on a plane that is three fourths of the way between the Gaussian image plane and the marginal image plane. However, based on diffraction, the optimum amount of defocus is different from the one found with geometric optics, and for small aberrations it is used to reduce the variance of the aberrations across the exit pupil of the lens. For small aberrations, as the Strehl ratio is maximum when the aberration variance is minimum, the best image plane is one that corresponds to the minimum variance. For small values of spherical aberration based

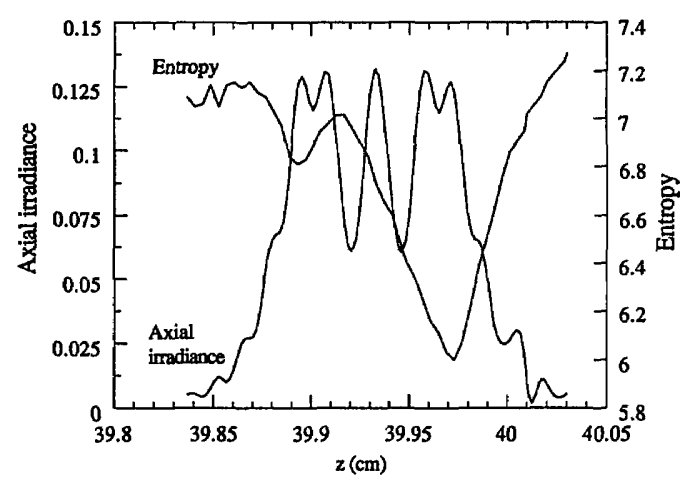

(a)

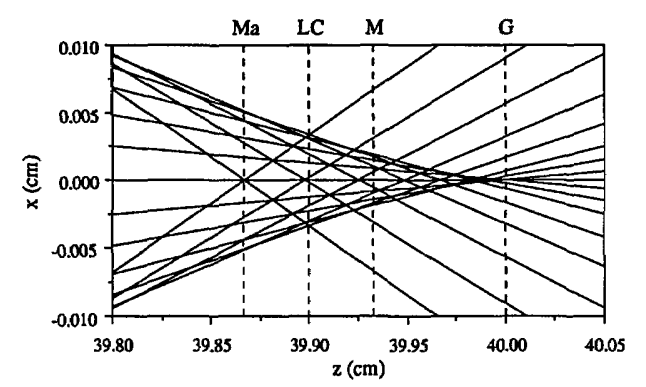

(b)

Fig. 2. (a) Entropy and axial irradiance for holographic lenses with a diameter of $8 \mathrm{~cm}$, (b) ray tracing (notation is the same as that of Fig. 1). 


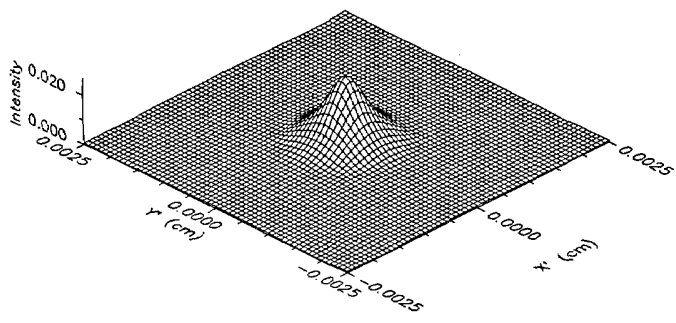

(a)

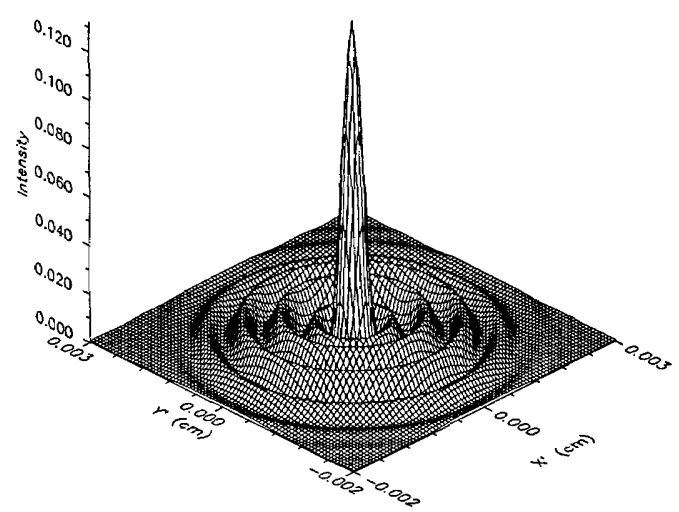

(b)

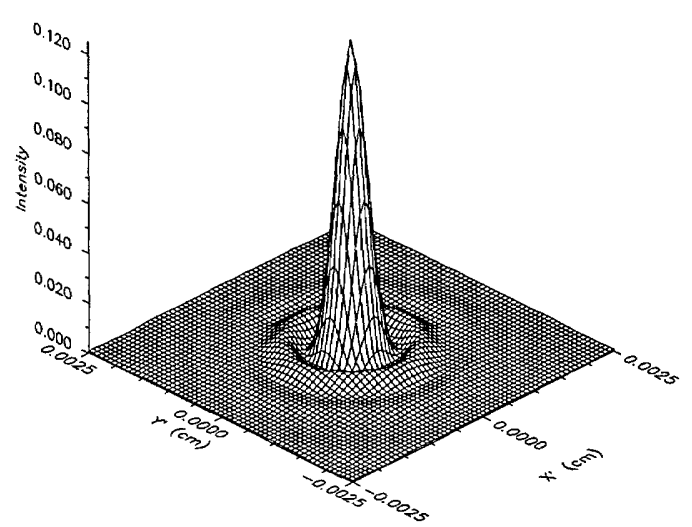

(c)

Fig. 3. Diffraction patterns for a lens diameter equal to $8 \mathrm{~cm}$ in (a) Gaussian plane (G), (b) the minimumaberration-variance plane (M), (c) the minimum-entropy plane.

on diffraction the best image is in a plane lying midway between the marginal and the Gaussian planes (the medium plane). This plane is different from the plane that contains the circle of least confusion of the geometric optics. The point of the best image plane, the one for which the irradiance is maximum, is the diffraction focus.

To obtain the entropy values, we chose the size of the integration plane by taking into account the values of the sizes of both the marginal and the paraxial planes and used the bigger of the two. Figure 1 shows the axial irradiance for the lens studied, the calculated entropy, and the ray tracing for the particular case in which the diameter lens is equal to
$5 \mathrm{~cm}$. The lowest value of the entropy and the maximum axial irradiance are at the same image plane, lying midway between the marginal and the Gaussian planes. The minimum-entropy plane corresponds in this case to the plane with minimum variance because spherical aberration is small. Figure 2 shows the axial irradiance, the entropy, and the ray tracing for different values of the $z$ position at the image plane when the diameter is $8 \mathrm{~cm}$. In this case the spherical aberration increases, and, as we can see from Fig. 2, there are different planes with maximum axial irradiance, but only one gives us minimum entropy, and it is different from the Gaussian plane and from the medium plane, where the aberration variance is minimum. There is a maximum value for the axial irradiance in the medium plane, but this plane does not have a minimum entropy value. Figure 3 shows the diffraction pattern for three different image planes: (a) the Gaussian plane, (b) the minimum-aberration-variance plane, and (c) the minimum-entropy plane, for the case of $D=8 \mathrm{~cm}$. As predicted, the best image plane in the sense of minimal aberrations is the minimum-entropy plane, and this plane does not coincide with medium plane $\mathrm{M}$, where the aberration variance is minimum. This happens because the hologram has large aberrations.

Summarizing, we have proposed a method for evaluating the image quality in HOE's, and the concept of entropy could be used to choose the best image plane in the sense of minimal aberrations. To illustrate our method we have shown some numerically evaluated examples for in-line holographic lenses when spherical aberration is present. The results shown in Figs. 2 and 3 support our initial suggestion for the use of the concept of entropy to locate the best image plane in HOE's. Finally, the study presented in this paper can also be extended to conventional optics.

This work was supported by the Direcció General d'Ensenyaments Universitaris i Investigació de la Generalitat Valenciana, Spain, project GV-1165/93.

\section{References}

1. R. Torroba, H. Rabal, and B. Ruiz, J. Mod. Opt. 39, 1939 (1992).

2. V. Michael Bove, J. Opt. Soc. Am. A 10, 561 (1993).

3. B. R. Frieden, J. Opt. Soc. Am. 62, 511 (1972).

4. B. R. Frieden and J. J. Burke, J. Opt. Soc. Am. 62, 1202 (1972).

5. E. H. Linfoot, J. Opt. Soc. Am. 45, 808 (1955).

6. E. L. O'Neill, Introduction to Statistical Optics (Dover, New York, 1991).

7. C. Dähne and F. Lanzl, Optik 55, 437 (1980).

8. B. R. Frieden, J. Mod. Opt. 35, 1297 (1988).

9. E. B. Champagne, J. Opt. Soc. Am. 57, 51 (1967).

10. A. Beléndez, L. Carretero, and A. Fimia, J. Opt. 22, 163 (1991).

11. J. N. Latta, Appl. Opt. 10, 599 (1971).

12. H. Chen, R. R. Hershey, and E. N. Leith, Appl. Opt. 26, 1983 (1987).

13. M. Born and E. Wolf, Principles of Optics (Pergamon, New York, 1987), p. 461. 